\title{
Comparative In Silico Molecular Docking Analysis of L-Threonine-3-Dehydrogenase, a Protein Target Against African Trypanosomiasis Using Selected Phytochemicals
}

\author{
Tehseen M. Dhorajiwala ${ }^{1}$, Sumit T. Halder ${ }^{1}$, Lalit Samant ${ }^{*}$ \\ ${ }^{1}$ Department of Bioinformatics, Patkar College, Goregaon West, Mumbai 400062, India \\ ${ }^{2}$ Molecular Genetics Research Laboratory, Scientific officer, Bai Jerbai Wadia Hospital for Children, Parel 400012, Maharashtra, \\ India
}

Corresponding Author: Lalit Samant, Mailing address: Molecular Genetics Research Laboratory, Bai Jerbai Wadia Hospital for Children, Parel 400012, Maharashtra, India. Tel: 022-24197346, E-mail: samantlalit@gmail.com

Received May 11, 2019; Accepted July 11, 2019; Online Published September 11, 2019

\begin{abstract}
Introduction: African trypanosomiasis is a neglected tropical disease caused by protozoa Trypanosoma brucei and transmitted via the bite of tsetse fly. The target protein of $T$. brucei is L-threonine-3-dehydrogenase, which has been selected for this study due to its metabolic importance for the parasite's survival. Docking of target protein was carried out with phytochemicals that passed in silico drug-likeness filters and have in vitro antitrypanosomal activity along with the standard drugs eflornithine and pentamidine available against the disease.

Materials and Methods: A 3D structure of L-threonine-3-dehyrogenase was downloaded from Protein Data Bank (PDB) with Id: 5K4Y and Pictorial database of 3D structures in the Protein Data Bank (PDBsum) was used to retrieve the active sites of the protein. The reviewed ligands were screened using SwissADME, Lipinski's rule of 5, and Molinspiration servers along with standard drugs and docked using AutoDock Vina and AutoDock 4.2.6. The 2D and 3D interacting residues were observed using Discovery Studio.

Results: Ligand Camptothecin which inhibited T. brucei during in vitro cytotoxic assays gave better binding affinity scores than the standard drugs (eflornithine and pentamidine) selected for this study. Camptothecin showed interaction with those active site residues where ligand NAD (nicotinamide-adenine-dinucleotide) binds to the target protein, which is a significant restricting pocket for the hindrance of the parasite.

Conclusions: Camptothecin derived from Camptotheca acuminata trees has the potential to be used as a better alternative than the standard drugs because of its less toxicity, better binding affinity, and specificity towards the inhibition of target protein.

Keywords: Docking Analysis, Phytochemicals, African Trypanosomiasis, Camptothecin

Citation: Dhorajiwala TM, Halder ST, Samant L. Comparative In silico molecular docking analysis of L-threonine-3-dehydrogenase, a protein target against African Trypanosomiasis using selected phytochemicals. J Appl Biotechnol Rep. 2019;6(3):101-108. doi:10.29252/JABR.06.03.04.
\end{abstract}

\section{Introduction}

African trypanosomiasis, when affecting humans, known as human African trypanosomiasis (HAT) is one of the most neglected tropical diseases having endemic status in Africa being widespread in over 37 countries in the continent. ${ }^{1}$ African trypanosomiasis also known as sleeping sickness is a parasitic disease transmitted through the bite of a vector fly named tsetse fly. The condition is classified as a neglected tropical disease by the World Health Organization (WHO), putting 65 million people at risk of developing the disease. ${ }^{2}$ This disease is mainly caused by 2 species of protozoa: Trypanosoma brucei gambiense and Trypanosoma brucei rhodesiense. About 98\% of the cases reported are caused by T. brucei gambiense, which creates a slow progression of the disease while the rest $2 \%$ are caused by T. brucei rhodesiense, which causes a more rapid form of the disease., ${ }^{2,3}$ The disease causes symptoms like fever, fatigue, weakness, weight loss, enlarged lymph nodes, cardiac dysfunction, endocrine disorders and progression to neurological symptoms in later stages of the disease. Symptoms affecting the nervous system include behavioral problems, motor disturbances, seizures, disturbances in the sleep cycle, insomnia, and optical problems. ${ }^{4}$ Medication for this disease is limited and poorly researched. The mechanism of action of prescribed drugs for HAT is still unknown, these drugs having severe side effects and also there is no vaccine developed against HAT. ${ }^{2}$ These conditions can be attributed to the fact that this disease mostly affects the countries with a more impoverished economy, leading to less funding for research and awareness by the governmental and healthcare agencies. $^{5}$

The protein target for the disease studied by Adjogatse et al led to the elucidation of the structure of the L-threonine3 -dehydrogenase protein from the protozoa T. brucei, the causative agent of African trypanosomiasis. This protein is a crucial enzyme in the synthesis of threonine which develops in the gut of the insect tsetse fly a vector for T. brucei. The

Copyright (C) 2019 The Author(s). This is an open-access article distributed under the terms of the Creative Commons Attribution License (http:// creativecommons.org/licenses/by/4.0), which permits unrestricted use, distribution, and reproduction in any medium, provided the original work is properly cited. 
inhibition of L-threonine-3-dehydrogenase causes the depletion of the parasite, as it is involved in the synthesis of the fatty acid pathway, energy production, and maintaining the stability of physiological processes. Another important reason for it to be a potential drug target is that L-threonine3-dehydrogenase is non-functional in human beings, thus making it a specific anti-parasitic target for potential lead candidates like phytochemicals. ${ }^{6}$ The conventional medicines prescribed against the disease include pentamidine, suramin, eflornithine, and melarsoprol. All of these standard drugs have potentially harmful effects on the body, and the exact mechanism of these drugs or which protein they target is still not clearly understood. Hence, there is a need for safer alternatives with fewer side effects, and thus phytochemicals should be considered for the treatment of this disease. ${ }^{3}$ Suramin is only administered through injection. It has various severe side effects which include cloudy urine, diarrhea, faintness, headache, itching, joint pain, swelling on skin, changes in or loss of vision, extreme tiredness or weakness, painful tender glands in the neck, armpits, or groin swelling around eyes. It is also considered to be a carcinogen, and little is known about the mechanism by which it kills the parasite or the protein it targets. ${ }^{7-9}$ Similarly, melarsoprol is also administered intravenously and is another acute toxic drug derived from arsenic, which is not even an acceptable compound group in docking. It has fatal effects such as encephalopathy. Around $5 \%$ of patients die while getting treated with melarsoprol.,10,11

Due to the many serious side-effects mentioned above, suramin and melarsoprol have not been considered to be taken as comparative drugs in this study. Pentamidine and eflornithine both administered through injections also have several side effects. These 2 drugs were chosen as relative standard drugs for the present study as they are less harmful than suramin and melarsoprol. ${ }^{7}$

Nowadays, the demand of natural products is increasing due to the abundant number of phytochemicals that can be obtained from a large selection of plants, herbs, etc. Within the last 25 years, a large proportion of developed drugs have had natural origins. A large number of studies have been carried out and have concluded that most of the natural products or phytochemicals have lead like properties or drug-like properties. ${ }^{12}$ In part 1 of prospective potential secondary metabolites against neglected tropical protozoan diseases, Schmidt et al conducted in vitro cytotoxic assays to determine phytochemicals with anti-trypanosomal activity. The phytochemicals which showed inhibition of the parasite included alloaromadendrene, cupacinosides from plant Cupania cinerea, lipiferolide from plant Liriodendron tulipifera, mukaadial and muzigadial from plant Warburgia ugandensis, pseudoguaianolide helenalin from Arnica and Helenium species, terpinen-4-ol, and $\alpha$-pinene. ${ }^{12}$ Similarly, in part 2 of the study Schmidt et al concluded that phytochemicals with the most inhibition activity against $T$. brucei based on the in vitro assay results were 2-(1-hydroxyethyl)-naphtho[2,3-b] furan-4,9-quinone, berberine, emetine, and quinidine. ${ }^{13}$ Hoet et al performed in vitro assays to determine potential antitrypanosomal phytochemicals. The most active compounds were Diospyrin from the bark of Diospyros montana,
O-methylmoschatoline from Unonopsis buchtienii, and pancracine from Narcissus angustifolius. ${ }^{14}$ Wink reviewed phytochemicals which had shown in vitro anti-trypanosomal activity, which included camptothecin from Camptotheca acuminata trees, carlina oxide from Carlina acaulis, sanguinarine, and vinblastine. ${ }^{15,16}$

The present study aimed to inhibit functionally important L-threonine-3-dehydrogenase protein through initiating the death of the parasite by docking it with the phytochemicals having anti- trypanosomal activity along with the standard drugs to give a better understanding of the active residues and the binding affinities by which they bind to the target protein.

\section{Materials and Methods}

Preparation of Target Protein

The target L-threonine-3-dehydrogenase protein from organism T. brucei, which is a causative agent for African trypanosomiasis was selected as the target protein for the study. The 3D structure of the target protein, retrieved from Protein Data Bank (PDB), has PDB Id: 5K4Y, and a resolution of $1.77 \AA .{ }^{17}$ The PDB database is an online repository for the crystallography structure of proteins and nucleic acids obtained by x-ray crystallography and NMR. ${ }^{18}$ After downloading the structure in PDB format it was visualized in the Discovery Studio Visualizer. ${ }^{19}$

Active Site Retrieval From Pictorial Database of 3D Structures in the Protein Data Bank (PDBsum)

The active sites of the target L-threonine-3-dehydrogenase protein from the organism T. brucei were retrieved using the PDBsum database. The PDBsum is a database having ligandprotein interaction information of those structures deposited in PDB. ${ }^{20}$ PDBsum gives information about the non-bonded and hydrogen bonded interactions of the ligand with the protein in the form of diagrams. The target protein PDB Id5K4Y was searched in PDBsum. The active sites for the target protein which is a homodimer were observed with 6 identical chains and 2 ligands (nicotinamide-adenine-dinucleotide [NAD] and acetate ion [ACT]) bonded in each chain. Chain F showed bonding with an addition ligand that was GOL (glycerol) along with NAD and ACT. ${ }^{21}$

The hydrogen-bonded interacting residues along with the non-bonded interactions were retrieved for chain $\mathrm{F}$ of the protein. Hence, chain $\mathrm{F}$ was used for the docking process after trimming the structure and deleting all the water molecules and heteroatoms.

\section{Energy Minimization and Protein Validation}

After the retrieval of the PDB protein structure and trimming it to retain its $\mathrm{F}$ chain, the protein was submitted on the Mod Refiner energy minimization server, which requires PDB input and afterwards the refined protein structure is mailed in PDB format to the user. ${ }^{22}$ The server refines the structure via 2 steps which include low resolution and high-resolution refinements of the protein. The first step builds central chain atoms using $\mathrm{C}$ alpha performing energy minimization followed by the second step which includes building side chain rotamers while refining the atoms again. ${ }^{23}$ 
The Ramachandran plot of the protein structure was checked before energy minimization using Rampage server. ${ }^{24}$ After energy minimization, the protein structure was downloaded from Modrefiner and uploaded on Rampage server for Ramachandran plot analysis. ${ }^{24}$ The improvement in protein structure was determined by analyzing the favored region, allowed region, and outliers in before and after energy minimized protein structures. ${ }^{24}$

Ramachandran plot is a way to understand the energetically allowed regions for backbone dihedral angles that are the psi vs phi of amino acid residues of a protein structure thus giving information about its stability and how it will fold. ${ }^{25}$

\section{Ligand Preparation}

Nineteen phytochemicals reviewed from literature studies having in vitro activity against African trypanosomiasis were selected along with the standard drugs (eflornithine and pentamidine). All the structures of the phytochemicals and drugs were drawn on MarvinSketch, which was followed by adding explicit hydrogen and cleaning the structures in $2 \mathrm{D}$ and $3 \mathrm{D}$. The structures were saved in SMILES and PDB formats. ${ }^{26}$

The ligands, along with standard drugs, were then submitted in SMILES format on SwissADME and Molinspiration servers. It was submitted in PDB format on SCFBio Lipinski Rule of Five server. ${ }^{27-29}$ SwissADME predicts the pharmacokinetic, ADME (absorption, distribution, metabolism, and excretion), physiochemical properties as well as whether a ligand has lead-likeness and drug-likeness features by subjecting the ligand to several parameters like molecular mass, number of hydrogen donors, acceptors, LogP, GI (gastrointestinal) absorption, solubility etc. ${ }^{27}$ Lipinski Rule of Five - SCFBio server predicts the rule of 5 properties for the submitted ligand. The Lipinski's rule of 5 helps to distinguish a drug-like compound from a non-drug on the basis of 5 rules. The molecule has a higher chance of having drug-likeness if it follows the following rules.

1. Molecular mass < $500 \mathrm{Da}$,

2. Lipophilicity $(\log \mathrm{P}<5)$,

3. Hydrogen bond donors $<5$

4. Hydrogen bond acceptors $<10$

5. Molar refractivity should be within 40 to $130 . .^{28}$

The Molinspiration server offers an online prediction of bioavailability scores for different parameters for the submitted ligand. The enzyme inhibitor parameter was evaluated for the phytochemicals and standard drugs since the target protein for the study is an enzyme. The server uses a distribution of activity scores of different ligands which include enzyme inhibitor, ion channel modulator, nuclear receptor ligand, etc. and compares their activity scores with the scores of 100000 drug-like molecules. This score allows the separation of active and inactive molecules. The higher the bioactivity score, the greater chance the molecule will be active and drug-like. ${ }^{30}$

\section{Docking Preparation}

In silico docking analysis of the target protein-ligand was carried out using AutoDock Vina and AutoDock 4.2.6. ${ }^{31-33}$ AutoDock Vina is designed for protein-ligand docking, utilizing multiple CPU's at a time, making it exceptionally faster and accurate. Vina is based on a gradient optimization algorithm along with local optimization and automatically clusters the results. ${ }^{31}$ The Molecular Graphics Lab (MGL) tools consists of: (a) Python Molecular Viewer (PMV), a software for structure visualization and $(b)$ AutoDock Tools (ADT), a software framework for structure editing and preparation of essential files required for docking. AutoDock Vina is a molecular docking program and is used to perform rigid-flexible type of docking. ${ }^{32,33}$

After loading the target protein in MGL tools, the water molecules were deleted, polar hydrogens were added, and the file was saved in pdbqt format. The active site residues of the target protein were then selected, and the grid box was made using 1 A spacing for the target protein. The ligand molecules in PDB format were loaded using AutoDock Tool's ligand menu, and all non-rotatable bonds were made rotatable, the ligand was then saved in pdbqt format as well. Docking was carried out using AutoDock Vina, by using the pdbqt files of the ligands, protein target and the configuration file containing dimensions of the grid box- that is centre $x, y, z$ and size $x, y, z$ values which were center_x $=44.325$, center_y $=273.658$, center $\_z=9.266$, size $\_x=28$, size $\_y=34$ and size $\_z=32$. The generated results were the out files giving the nine poses of the ligand docked with the target protein in pdbqt format and a log file that gave information about the binding energy and RMSD values by which the ligand binds to the target protein.

AutoDock 4.2.6 is a docking program based on Lamarckian genetic algorithm and semi-empirical free energy force field that generates free binding energy of 10 ligand poses after docking. ${ }^{33}$ The grid box dimensions were kept the same while docking using AutoDock 4.2.6, to validate the docking results produced by using AutoDock Vina.

The protein file was loaded in MGL tools, the water molecules were deleted, and polar hydrogen was added using edit option, and the target protein were saved in pdbqt format. The grid box around the active residues was made using the AutoDock tools, keeping the same grid box dimensions and grid coordinates that were used in AutoDock Vina. The grid parameter file (gpf) was saved from the output option of the grid menu. The ligands were loaded from the ligand menu of AutoDock tools, and then from the docking menu, the receptor was chosen and the ligand file was chosen. The docking parameter files (dpfs) were saved from the output option of the docking menu by choosing the Lamarckian genetic algorithm. After the generation of gpf and dpf files, Autogrid was run followed by AutoDock 4 from the docking menu. The docked dlg file produced the output of 10 ligand poses, which was analyzed from the analyze menu of AutoDock tools and was saved in pdbqt format to further analyze it in the Discovery Studio Visualizer.

The 2D and 3D protein-ligand interactions were visualized in Discovery Studio Visualizer. ${ }^{19}$

\section{Results}

L-Threonine-3-Dehydrogenase Protein

After downloading and trimming the protein L-threonine-3- 
dehydrogenase, PDB Id- 5K4Y to its F chain, the protein was visualized in the Discovery Studio Visualizer. Figure 1 shows the visualized $\mathrm{F}$ chain of L-threonine-3-dehydrogenase.

Energy Minimization and Protein Validation by Using Rampage Server

After downloading the refined structure mailed by Mod Refiner server, the before and after minimized structure was validated on the Rampage server to check the allowed regions, favored region, and outliers (Table 1).

After retrieval of L-threonine-3-dehydrogenase from PDB and its energy minimization, the validation was performed by checking the Ramachandran plot. The allowed and favored regions of the protein structure was already in the acceptable range (98\% and 2\%). After energy minimization, these percentages remained the same. The aim of minimization was to eliminate leucine from the outlier region. After energy minimization, the outlier residue changed from leucine to proline. Proline and glycine residues do not follow the Ramachandran plot. So having these residues as an outlier is acceptable as it will not affect the final results. ${ }^{34}$ Thus carrying out the energy minimization step improved the overall structure for the docking analysis of the protein.

\section{Active Site Results}

The 3 ligands present on the F chain which are NAD, ACT and GOL were checked for their interacting $\mathrm{H}$ bonds and nonbonded interactions with the target protein using PDBsum database (Table 2).

Being a PDB deposited structure, the PDBsum database was used for retrieving the active site residue numbers (Table 2).

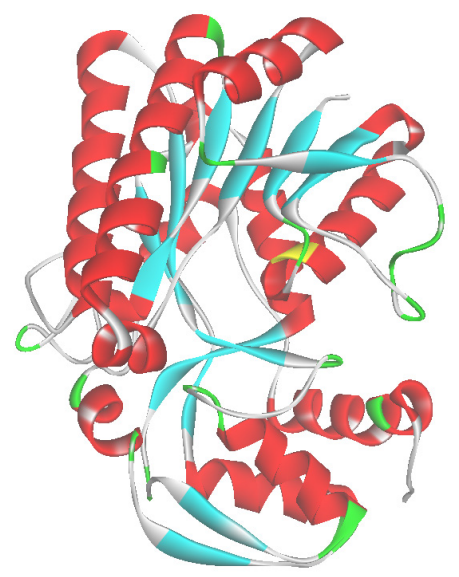

Figure 1. Chain F Trimmed Protein L-Threonine-3-Dehydrogenase PDB Id- 5K4Y in Discovery Studio Visualizer.

Table 1. Ramachandran Results for Before and After Energy Minimization of Protein L-Threonine-3-Dehydrogenase Using ModRefiner Server

\begin{tabular}{lll}
\hline Ramachandran Results & $\begin{array}{l}\text { Before Energy } \\
\text { Minimization }\end{array}$ & $\begin{array}{l}\text { After Energy } \\
\text { Minimization }\end{array}$ \\
\hline Number of allowed regions & $98.3 \%$ & $98.4 \%$ \\
Number of favored regions & $1.3 \%$ & $1.3 \%$ \\
\multirow{2}{*}{ Number of outliers } & $\begin{array}{l}0.3 \%(1 \text { residue - } \\
\text { Leucine) }\end{array}$ & $\begin{array}{l}0.3 \%(1 \text { residue- } \\
\text { Proline) }\end{array}$ \\
\hline
\end{tabular}

The non-bonded interaction of the ligands NAD, ACT, GOL with the protein along with the hydrogen interactions were retrieved to be further used in the docking process.

Hydrogen bonds are the most crucial bonds which need to be considered while searching for active sites, mainly because $\mathrm{H}$ bonds are the most essential contributors in the stability of the protein structure. This is due to the fact that proteins are made of $\mathrm{NH}$ and $\mathrm{OH}$ groups that can donate $\mathrm{H}$ bonds and other groups that will accept them. Thus, $\mathrm{H}$ bonds help in the specificity of protein-ligand interactions stabilizing the ligand in the binding pockets. ${ }^{35}$

SwissADME Results for Standard Drugs and Phytochemicals The 19 phytochemicals having activity against African trypanosomiasis along with standard drugs used for the treatment of African trypanosomiasis were submitted to the SwissADME server to check whether they had potential properties to be a drug or not. Among them, 7 ligands passed the server (Table 3).

After submitting 19 ligands, 6 ligands passed and showed no violations in SwissADME filters and were predicted to be having drug-like properties. These filters compare the structure to a particular range of physiochemical properties. These properties vary from one filter to others to predict it to be drug-like. ${ }^{36}$ The standard drugs prescribed against the African trypanosomiasis, including eflornithine \& pentamidine, failed to pass on SwissADME (Table 3) . Eflornithine failed to be predicted as a drug-like violating Ghose and Muegge filter present in SwissADME. The drug gave one violation of the Ghose filter by having molar refractivity less than 40 , and gave 2 violations of the Muegge filter by having a molecular weight less than $200 \mathrm{~g}$, XLOGP3 <-2. It was predicted to be a not lead-like compound as it violated lead likeness filter with a molecular weight less than $200 \mathrm{~g}$. Pentamidine was predicted as an unfit compound to be a lead molecule by violating lead likeness filter by having less than 7 rotors.

\section{SCFBio Lipinski Rule of 5 Server Result}

The 7 ligands which passed the SwissADME server filters and were predicted drug-like by the server were submitted on SCFBio Lipinski's rule of 5 server, to check if ligands and drugs pass the Lipinski's rule of 5 . The results have been presented in Table 4.

All the 7 ligands, along with the standard drugs, passed the Lipinski's rule of 5 server.

Table 2. Active Sites Obtained Using PDBsum for L-Threonine-3-Dehydrogenase Protein

\begin{tabular}{ll}
\hline $\begin{array}{l}\text { Ligands Present } \\
\text { on F Chain }\end{array}$ & Active Site Residue Number \\
\hline NAD & $\begin{array}{l}6009,6011,6012,6013,6014,6035,6036,6054,6055, \\
6056,6077,6078,6079,6081,6095,6117,6119,6144, \\
6148,6171,6172,6173,6174\end{array}$ \\
ACT & $6081,6119,6120,6144,6280$ \\
GOL & $6017,6176,6177,6178,6216,6217,6218$ \\
\hline
\end{tabular}

Abbreviations: NAD, nicotinamide-adenine-dinucleotide; ACT, acetate ion; GOL, glycerol. 
Table 3. SwissADME Results for Standard Drugs and Ligands

\begin{tabular}{|c|c|c|c|c|c|c|}
\hline \multirow{3}{*}{ Drug/Ligand Name } & \multicolumn{6}{|c|}{ Filters } \\
\hline & \multicolumn{2}{|c|}{ Physiochemical Properties } & \multirow{2}{*}{$\begin{array}{c}\text { Lipophilicity } \\
\text { Consensus Log P }\end{array}$} & \multirow{2}{*}{$\begin{array}{c}\text { Pharmacokinetics } \\
\text { GI Absorption }\end{array}$} & \multirow{2}{*}{ Water Solubility } & \multirow{2}{*}{ Lead Likeness } \\
\hline & TPSA $\left(\AA^{2}\right)$ & No. of rotatable bonds & & & & \\
\hline Pentamidine & 118.20 & 10 & 2.72 & High & Moderately Soluble & No \\
\hline Camptothecin & 81.42 & 1 & 2.20 & High & Soluble & Yes \\
\hline Lipiferolide & 65.13 & 2 & 2.33 & High & Soluble & Yes \\
\hline Mukaadial & 74.60 & 2 & 1.49 & High & Soluble & Yes \\
\hline Muzigadial & 49.69 & 0 & 1.94 & High & Soluble & Yes \\
\hline O-Methylmoschatoline & 57.65 & 3 & 3.02 & High & Moderately Soluble & Yes \\
\hline Pancracine & 62.16 & 0 & 0.92 & High & Very Soluble & Yes \\
\hline Quinidine & 45.59 & 4 & 2.76 & High & Soluble & Yes \\
\hline
\end{tabular}

Note: TPSA ( $\AA 2$ ) should be less than 140; Consensus Log p should be less than 5; Rotatable bond should be less than 7.

Table 4. Lipinski's Rule of 5 Results for the Standard Drugs and Ligands

\begin{tabular}{|c|c|c|c|c|c|}
\hline \multirow{2}{*}{ Drug/Ligand Name } & \multicolumn{5}{|c|}{ Property } \\
\hline & Mass, mg/mol & H Acceptor & H Donor & $\log P$ & Refractivity \\
\hline Eflornithine & 182.00 & 4 & 5 & -0.227499 & 38.846592 \\
\hline Camptothecin & 348.00 & 6 & 1 & 1.6933 & 92.562775 \\
\hline Lipiferolide & 306.00 & 5 & 0 & 2.3036 & 78.949974 \\
\hline Mukaadial & 266.00 & 4 & 2 & 1.2488 & 70.396576 \\
\hline Muzigadial & 250.00 & 3 & 2 & 2.0048 & 68.743576 \\
\hline O-Methylmoschatoline & 321.00 & 5 & 0 & 3.3409099 & 91.435478 \\
\hline Pancracine & 287.00 & 5 & 2 & 0.7486 & 74.217575 \\
\hline Quinidine & 324.00 & 4 & 1 & 3.1731 & 95.026764 \\
\hline
\end{tabular}

Molinspiration Results for Standard Drugs and Ligands The 7 ligands which passed both the SwissADME and SCFBio's Lipinski rule of 5 servers along with the 2 standard drugs were submitted on the Molinspiration server to check if the ligands had properties to be an enzyme inhibitor (Table 5).

Six ligands passed the enzyme filter and were predicted to be enzyme inhibitors with camptothecin having the highest score among the ligands. The larger the value of the score, the more probability that the ligand will be active. Since camptothecin has a score of 1.11 , it has the highest probability of being an active enzyme inhibitor. Both drugs were predicted as non-enzyme inhibitors. The target protein of the study is an enzyme; hence the prediction of this filter is an important parameter. These 6 ligands were further used for the docking

Table 5. Molinspiration Results for Standard Drugs and Ligands

\begin{tabular}{lcc}
\hline Drug /Ligand Name & Enzyme Inhibitor & Bioactivity Score \\
\hline Eflornithine & No & -0.08 \\
\hline Pentamidine & No & 0.16 \\
\hline Camptothecin & Yes & 1.11 \\
\hline Lipiferolide & Yes & 0.99 \\
Mukaadial & Yes & 0.69 \\
\hline Muzigadial & Yes & 0.72 \\
\hline O-methylmoschatoline & Yes & 0.41 \\
\hline Pancracine & Yes & 0.37 \\
\hline Quinidine & No & 0.11 \\
\hline
\end{tabular}

analysis with the target protein.

AutoDock Vina \& AutoDock 4.2.6 Results

AutoDock Vina and AutoDock 4.2.6 were used to dock the 6 ligands with the target protein. The results of the highest scoring ligand along with standard drugs are summarized in Tables 6 and 7.

Camptothecin showed highest binding affinity using both Autodock Vina and Autodock 4.2.6 than the standard drugs (eflornithine and pentamidine).

Table 6. AutoDock Vina Docking Results- Binding Energy Values for Standard Drugs and Ligand Docked With Target Protein L-Threonine-3-Dehydrogenase, a Target Against African Trypanosomiasis

\begin{tabular}{lc}
\hline Drug/Ligand Name & Binding Energy (kcal/mol) \\
\hline Eflornithine & -4.6 \\
Pentamidine & -7.4 \\
Camptothecin & -8.4 \\
\hline
\end{tabular}

Table 7. AutoDock 4.2.6 Docking Results- Binding Energy Values for Standard Drugs and Ligand Docked With Target Protein L-Threonine-3-Dehydrogenase, a Target Against African Trypanosomiasis

\begin{tabular}{lc}
\hline Drug/Ligand Name & Binding Energy $(\mathbf{k c a l} / \mathbf{m o l})$ \\
\hline Eflornithine & -5.16 \\
Pentamidine & -3.05 \\
Camptothecin & -5.58 \\
\hline
\end{tabular}




\section{Discussion}

African trypanosomiasis has been considered to be one the most neglected diseases in the world as there are limited treatment options available with detrimental side effects. Hence, safer and better alternatives need to be studied to treat this disease. All the 4 drugs available for treatment of this disease are only administered through IV route and there is no oral medication available for this disease. Natural drugbased therapy needs to be researched and studied against this disease which was the main aim of this study.

The L-threonine-3-dehydrogenase is an important functioning enzyme of the parasite T. brucei. Its structure and important catalytic activity site was elucidated by Adjogatse et al. This study explained the significance of the ligand NAD's binding pocket in the protein as an important catalytic site which can be targeted for structure-based drug studies in order to block the pathway of the enzyme L-threonine-3dehydrogenase. This enzyme has many important functions in the metabolism and stability of the parasite. Blocking the particular NAD binding pocket as described in the study will bring the death of the parasite. ${ }^{6}$

Seven out of 20 ligands passing the SwissADME and Lipinski's rule of 5 servers were checked for their enzyme inhibition activity. The bioactivity score of the enzyme inhibition was predicted by the Molinspiration server for the ligands and the drugs. Six ligands passed the enzyme filter, indicating that they had enzyme inhibition activity while the drugs failed. As the target protein is an enzyme, the prediction of this filter was an important parameter for the study. These 6 ligands had been studied in-vitro to determine their activity against the inhibition of T. brucei.

Camptothecin, obtained in alcoholic extracts of $C$. acuminata trees were studied in vitro in a cytotoxicity assay to test the inhibition of T. brucei by Bodley et al. The results of Bodley et al's study proved that camptothecin is lethal to T. brucei with EC50 values of $1.5 \mu \mathrm{M}$. These parasites were completely eliminated at high concentrations of camptothecin. Also, these levels are within the range for other anti-trypanosomal drugs in the assay (e.g., 0.02 and $22 \mu \mathrm{M}$ for pentamidine and eflornithine, respectively). ${ }^{15,16}$

Lipiferolide isolated from Liriodendron tulipifera showed to be the most active and selective parthenolide derivative against T. brucei found so far with an IC50 of $0.22 \mu \mathrm{M}$. Muzigadial and mukaadial isolated from Warburgia ugandensis showed inhibition of T. brucei with IC50 values of 0.56 and $0.64 \mu \mathrm{M}$, respectively. ${ }^{12}$

O-methylmoschatoline isolated from the stem bark of Unonopsis buchtienii showed inhibition activity on the $T$. brucei $(\mathrm{IC} 100=20 \mu \mathrm{M})$ but without selectivity. Pancracine, an alkaloid obtained from the fresh bulb of Narcissus angustifolius subsp. transcarpathicus was active on the mammalian stage of T. brucei with an IC50 of $2.4 \mu \mathrm{M} .{ }^{14}$

The L-threonine-3-dehydrogenase protein was docked with the 5 ligands and the 2 standard drugs with AutoDock Vina and AutoDock 4.2.6 using the same grid box dimensions in-order to compare and validate the docking results. The output of the docking was the free binding energy with which the ligand binds to the pocket of the receptor protein. The highest scoring ligand (camptothecin) gave a better binding affinity score of -8.4 using AutoDock Vina and -5.58 using AutoDock 4.2.6 than the 2 standard drugs (eflornithine and pentamidine).

The standard drugs showed unfavorable bonds after docking in AutoDock Vina. The 2D interactions are shown in Figure $2 \mathrm{~A}$ and Figure $3 \mathrm{~A}$, and the $3 \mathrm{D}$ interactions are shown in Figure $2 \mathrm{~B}$ and Figure $3 \mathrm{~B}$.

Eflornithine showed unfavorable bonds after docking in AutoDock 4.2.6, while pentamidine did not form any unfavorable bond. The $2 \mathrm{D}$ interactions of the drugs are shown in Figure 5A and Figure 6A, and the 3D interactions are shown in Figure $5 \mathrm{~B}$ and Figure 6B.

Unfavorable bonds affect the activity stability of the drug. The formation of any kind of unfavorable bond between/in protein-ligand complex reduces the stability of the complex as these types of bonds indicate a force of repulsion occurring between 2 molecules and an atom.

Camptothecin formed conventional hydrogen bonds along with other types of interactions with the target protein. In AutoDock Vina, camptothecin formed conventional hydrogen
A

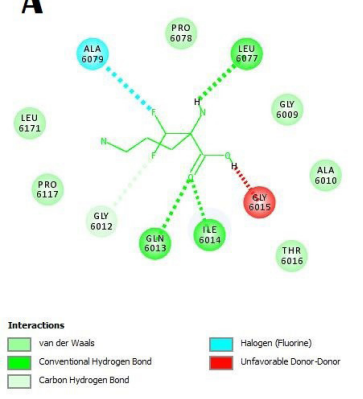

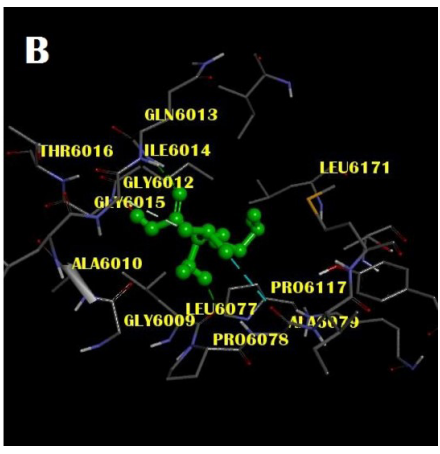

Figure 2. (A) 2D interaction of drug eflornithine with target protein docked using AutoDock Vina, visualized in Discovery Studio, residue glycine 6015 forming unfavorable Donor-Donor interaction represented in red dotted lines. (B) 3D interaction of drug eflornithine with the target protein docked using AutoDock Vina, the ligand represented in ball and stick format in green, interacting residues labeled in yellow.
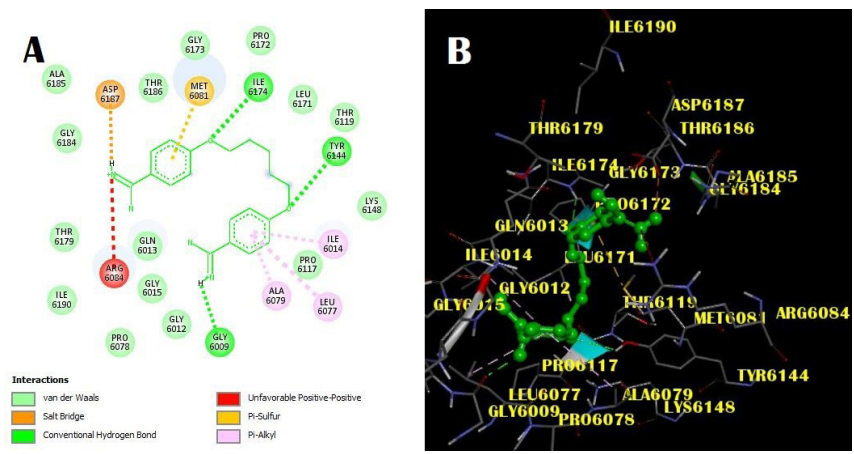

Figure 3. (A) 2D interaction of drug pentamidine with target protein docked using AutoDock Vina, visualized in Discovery Studio, arginine 6084 forming unfavorable positive-positive interaction represented in red dotted lines. (B) 3D interaction of drug pentamidine with the target protein docked using AutoDock Vina, the ligand represented in ball and stick format in green, interacting residues labeled in yellow. 


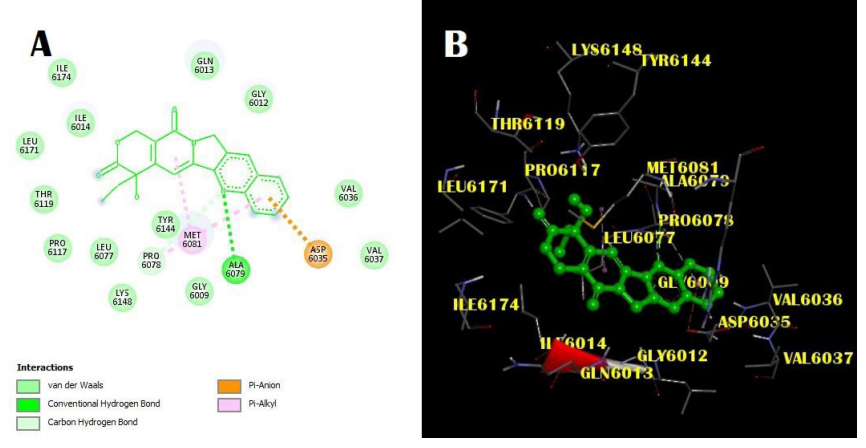

Figure 4. (A) 2D interaction of ligand camptothecin with target protein docked using AutoDock Vina, visualized in Discovery Studio, alanine 6079 forming conventional Hydrogen bonds represented in green dotted lines. (B) 3D interaction of ligand camptothecin with the target protein docked using AutoDock Vina, the ligand camptothecin represented in ball and stick format in green, interacting residues labeled in yellow.

A

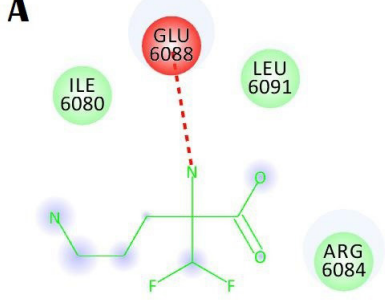

$\square$ ven der Woads
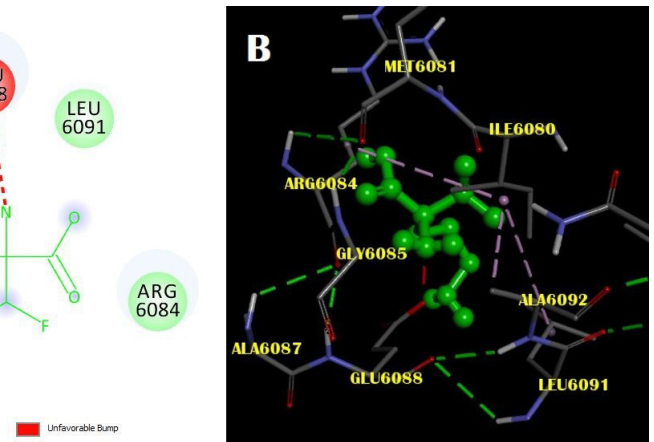

Figure 5. (A) 2D interaction of drug eflornithine with target protein docked using AutoDock 4.2.6, visualized in Discovery Studio, residue glutamate 6088 forming unfavorable bump interaction shown in red dotted line. (B) 3D interaction of drug eflornithine with the target protein docked using AutoDock 4.2.6, the ligand represented in ball and stick format in green, interacting residues labeled in yellow.

bonds with residue alanine 6079 , as shown in a $2 \mathrm{D}$ interaction in Figure 4A and a 3D interaction in Figure 4B. In AutoDock 4.2.6, camptothecin formed conventional hydrogen bonds with residue alanine 6079 and glycine 6013 , as shown in a 2D interaction Figure 7A and a 3D interaction in Figure 7B. This proves that ligand has the capability of forming conventional hydrogen bonds that have great significance in the stability of protein-ligand complex with better binding affinity than the standard drugs.

After docking in AutoDock Vina and AutoDock 4.2.6, camptothecin was found bonded to the common amino acid residues including glycine 6009 , glycine 6012, glycine 6013 , isoleucine 6014 , aspartate 6035 , leucine 6077 , tyrosine 6144 , lysine 6148 , alanine 6036 , leucine 6077 , proline 6078 , methionine 6081, proline 6117, threonine 6119 and leucine 6171. These residues are from the important active pocket as mentioned in the study where the ligand NAD binds, and camptothecin binding to the same residues in the pocket proves it can inhibit the parasite.

Adjogatse et al concluded that the NAD binding pocket of protein L-threonine-3-dehydrogenase is a deep and large

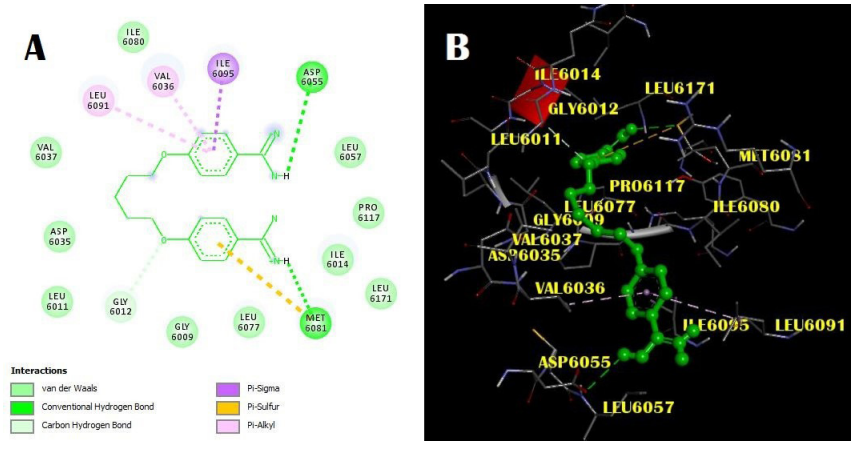

Figure 6. (A) 2D interaction of drug pentamidine with target protein docked using AutoDock 4.2.6, visualized in Discovery Studio, residue aspartate 6055 and methionine 6081 forming conventional hydrogen bonds represented in green dotted lines. (B) 3D interaction of drug pentamidine with the target protein docked using AutoDock 4.2.6, the ligand represented in ball and stick format in green, interacting residues labeled in yellow.

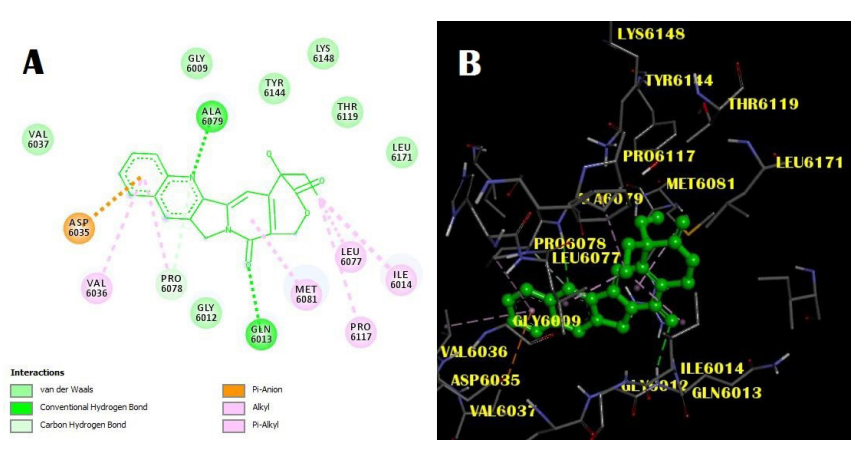

Figure 7. (A) 2D interaction of ligand camptothecin with target protein docked using AutoDock 4.2.6, visualized in Discovery Studio. Glycine 6013 and alanine 6079 forming conventional hydrogen bonds represented in green dotted lines. (B) 3D interaction of camptothecin with the target protein docked using AutoDock 4.2.6, the ligand represented in ball and stick format in green color, interacting residues labeled in yellow color. binding pocket where many important active residues are present which are hydrophobic and hydrophilic in nature and thus should be targeted for designing highly specific inhibitors. $^{6}$

In silico toxicity screening and docking studies proved that camptothecin has better binding affinity than the standard drugs and also binds to the functionally important active site of the target protein as predicted by both AutoDock Vina and Autodock 4.2.6 thus validating the results.

African trypanosomiasis has been plaguing mankind since centuries, affecting millions and still putting many people at risk of developing the disease. No better treatment option has yet been developed for those suffering from it. According to the results of this study, camptothecin has proved to have a potential to be a natural inhibitor of the parasite against African trypanosomiasis.

\section{Conclusions}

The metabolic pathway of the protozoa is dependent on the protein L-threonine-3-dehydrogenase. An important active site for the inhibition of the protein target is where 
ligand NAD binds as understood from experimental studies of the protein structure. Docking the protein target with the screened phytochemicals having passed the SwissADME filter, Lipinski's rule of 5 and Molinspiration server's enzyme inhibition filter, led to 5 compounds.

Among the 6 ligands, camptothecin showed a better binding affinity for the target protein even performing better than the standard drugs (eflornithine and pentamidine). It actually binded to the same amino acid residues as ligand NAD does which is an important active pocket of the protein. Being a phytochemical, the ligand has no side effects as they are naturally derived compounds. The ligand camptothecin has been studied in-vitro in cytotoxic assays inhibiting $T$. brucei. This study proved that camptothecin derived from $C$. acuminata trees are a natural inhibitor of the parasite.

\section{Authors' Contributions}

All authors contributed equally to this research.

\section{Conflict of Interest Disclosures}

The authors declare that there is no conflict of interest regarding the publication of this article.

\section{Acknowledgments}

Authors sincerely thank Molecular Genetics Research lab staff members and B. J. Wadia Hospital for Children, for providing facility and necessary effort for this research.

\section{References}

1. Global Health Observatory (GHO) data. https://www.who.int/gho/ neglected_diseases/human_african_trypanosomiasis/en/.

2. Trypanosomiasis, human African (sleeping sickness). https://www. who.int/news-room/fact-sheets/detail/trypanosomiasis-humanafrican-(sleeping-sickness).

3. Büscher P, Cecchi G, Jamonneau V, Priotto G. Human African trypanosomiasis. Lancet. 2017;390(10110):2397-2409. doi:10.1016/s0140-6736(17)31510-6.

4. Kennedy PG. Clinical features, diagnosis, and treatment of human African trypanosomiasis (sleeping sickness). Lancet Neurol. 2013;12(2):186-194. doi:10.1016/s1474-4422(12)70296-x.

5. Aksoy S, Buscher P, Lehane M, Solano P, Van Den Abbeele J. Human African trypanosomiasis control: Achievements and challenges. PLoS Negl Trop Dis. 2017;11(4):e0005454. doi:10.1371/journal. pntd.0005454.

6. Adjogatse E, Erskine P, Wells SA, et al. Structure and function of L-threonine-3-dehydrogenase from the parasitic protozoan Trypanosoma brucei revealed by X-ray crystallography and geometric simulations. Acta Crystallogr D Struct Biol. 2018;74(Pt 9):861-876. doi:10.1107/s2059798318009208.

7. Resources for Health Professionals. https://www.cdc.gov/parasites/ sleepingsickness/health_professionals/index.html.

8. Suramin (Injection route). https://web.archive.org/ web/20170908214205/https://www.ncbi.nlm.nih.gov/pubmedhealth/PMH0045224/.

9. Suramin. https://www.drugbank.ca/drugs/DB04786.

10. WHO Model Prescribing Information: Drugs Used in Parasitic Diseases. 2nd ed. Melarsoprol. http://apps.who.int/medicinedocs/ en/d/Jh2922e/2.10.3.html\#Jh2922e.2.10.3.

11. Melarsoprol. PubChem; 2019. https://pubchem.ncbi.nlm.nih.gov/ compound/Melarsoprol.

12. SchmidtTJ, Khalid SA, Romanha AJ, et al. The potential of secondary metabolites from plants as drugs or leads against protozoan neglected diseases - part I. Curr Med Chem. 2012;19(14):21282175. doi:10.2174/092986712800229023.

13. SchmidtTJ, Khalid SA, Romanha AJ, et al. The potential of secondary metabolites from plants as drugs or leads against protozoan neglected diseases - part II. Curr Med Chem. 2012;19(14):21762228. doi:10.2174/092986712800229087.

14. Hoet S, Opperdoes F, Brun R, Quetin-Leclercq J. Natural products active against African trypanosomes: a step towards new drugs. Nat Prod Rep. 2004;21(3):353-364. doi:10.1039/b311021b.

15. Wink M. Medicinal plants: a source of anti-parasitic secondary metabolites. Molecules. 2012;17(11):12771-12791. doi:10.3390/ molecules171112771.

16. Bodley AL, Shapiro TA. Molecular and cytotoxic effects of camptothecin, a topoisomerase I inhibitor, on trypanosomes and Leishmania. Proc Natl Acad Sci U S A. 1995;92(9):3726-3730. doi:10.1073/pnas.92.9.3726.

17. Adjogatse EA, Erskine PT, Cooper JB. 5K4Y. 2018. https://www. rcsb.org/structure/5K4Y.

18. Berman HM, Westbrook J, Feng Z, et al. The Protein Data Bank. Nucleic Acids Res. 2000;28(1):235-242. doi:10.1093/ nar/28.1.235.

19. Discovery Studio Visualizer. San Diego: Dassault Systèmes BIOVIA; 2017. https://www.3dsbiovia.com/products/collaborative-science/ biovia-discovery-studio/.

20. PDBsum Pictorial database of $3 \mathrm{D}$ structures in the Protein Data Bank. EMBL-EBI; 2013. http://www.ebi.ac.uk/thornton-srv/ databases/cgi-bin/pdbsum/GetPage.pl?pdbcode=index.html.

21. About PDBsum. EMBL-EBI. 2013. http://www.ebi.ac.uk/ thornton-srv/databases/cgi-bin/pdbsum/GetPage.pl?pdbcode=n/ a\&template=doc_about.html.

22. ModRefiner. Zhang Lab. 2018. https://zhanglab.ccmb.med.umich. edu/ModRefiner/.

23. $\mathrm{Xu} \mathrm{D,} \mathrm{Zhang} \mathrm{Y.} \mathrm{Improving} \mathrm{the} \mathrm{physical} \mathrm{realism} \mathrm{and} \mathrm{structural}$ accuracy of protein models by a two-step atomic-level energy minimization. Biophys J. 2011;101(10):2525-2534. doi:10.1016/j. bpj.2011.10.024.

24. RAMPAGE. http://mordred.bioc.cam.ac.uk/ rapper/rampage.php.

25. Ramachandran GN, Ramakrishnan C, Sasisekharan V. Stereochemistry of polypeptide chain configurations. J Mol Biol. 1963;7:95-99. doi:10.1016/s0022-2836(63)80023-6.

26. MarvinSketch. Budapest, Hungary: ChemAxon; 2017. https:// chemaxon.com/products/marvin.

27. Daina A, Michielin O, Zoete V. SwissADME: a free web tool to evaluate pharmacokinetics, drug-likeness and medicinal chemistry friendliness of small molecules. Sci Rep. 2017;7:42717. doi:10.1038/srep42717.

28. Lipinski CA. Lead- and drug-like compounds: the rule-of-five revolution. Drug Discov Today Technol. 2004;1(4):337-341. doi:10.1016/j.ddtec.2004.11.007.

29. Molinspiration. Slovensky Grob, Slovakia Republic: Bratislava University; 2019. https://www.molinspiration.com/cgi-bin/ properties.

30. Molinspiration drug-likeness \& bioactivity score. Bratislava University; 2002. https://www.molinspiration.com/docu/miscreen/ druglikeness.html.

31. Trott O, Olson AJ. AutoDock Vina: improving the speed and accuracy of docking with a new scoring function, efficient optimization, and multithreading. J Comput Chem. 2010;31(2):455-461. doi:10.1002/jcc.21334.

32. Sanner MF. Python: a programming language for software integration and development. J Mol Graph Model. 1999;17(1):5761.

33. Morris GM, Huey R, Lindstrom W, et al. AutoDock4 and AutoDockTools4: Automated docking with selective receptor flexibility. J Comput Chem. 2009;30(16):2785-2791. doi:10.1002/ jcc. 21256.

34. Lovell SC, Davis IW, Arendall WB, 3rd, et al. Structure validation by Calpha geometry: phi, psi and Cbeta deviation. Proteins. 2003;50(3):437-450. doi:10.1002/prot.10286.

35. Hydrogen Bond. Elsevier Publications; 2018. https://www. sciencedirect.com/topics/biochemistry-genetics-and-molecularbiology/hydrogen-bond.

36. SwissADME-Frequently Asked Questions. SIB; 2019. http://www. swissadme.ch/faq.php. 